\title{
A new pulsating white dwarf in the Wide Field Camera?
}

\author{
D. Wonnacott, \\ Rutherford Appleton Laboratory, Chilton, Didcot, Oxon., OX11 OQX, UK.
}

\begin{abstract}
Recent observations in the extreme ultraviolet have shown that the binary companion of the pulsating metallic-lined A8m: star IK Peg is a hot DA white dwarf. The EUV light curve is shown to be subject to too much photon noise to identify any periods, and simulations of the effect of a hotspot on the compact object indicate that it is unlikely that the observed photometric variability is not intrinsic to the $\mathrm{A} 8 \mathrm{~m}$ : star.
\end{abstract}

Given that there are more pulsating white dwarfs in the Galaxy than $\delta$ Scuti stars, this EUV detection warrants investigation to see if the pulsations ascribed to the Amstar can, in fact, be attributed to hot spots on a rotating white dwarf or accretion from the ISM even though the magnitude difference is $\sim 7^{\mathrm{m}}$.

Firstly, the WFC EUV light-curves for the two filter passbands $(100 \AA$ and $137 \AA$ respectively for S1a and S2a) were subjected to a period analysis using the technique due to Scargle (1982). The periodograms of the data are very sensitive to the photon noise induced by the low count rates and short observation slots $(\sim 40-80 \mathrm{~s})$. A comparison of the periodograms yields no coincident peaks making it unlikely that there is a real (detectable) period present.

As a further test, the times of the original observations of IK Peg (Kurtz 1978) were used to generate a series of 'observations' by sampling a single-frequency sine wave at those same times. As the measurements were nearly evenly spaced $(\Delta t \sim$ $0.005 \sim 7{ }^{\mathrm{m}} 2$ ), there is a strong possibility that a such an signal would be contaminated by beating with the observation period. These 'light-curves' were subjected to the same period analysis as above and compared with an identical analysis of the original $y$-band photometry. White dwarf variations were simulated in the range 2-25 minutes and for various phases relative to the first observation.

Examination of the power spectra of these light-curves, however, clearly shows that there is insufficient leakage of power from the white dwarf-like (high) frequencies to produce a dominant 23.9 cycles/day beat. This is true of all the high frequencies investigated. As the observed frequency is too low to be accounted for by a pulsating or rotating white dwarf, it can therefore only be attributed to the metallic-lined star and its apparently contradictory properties.

\section{References:}

Scargle J. D., $A p . J ., 1980.263,835$.

Kurtz D., 1978. Ap. J., 221, 869. 